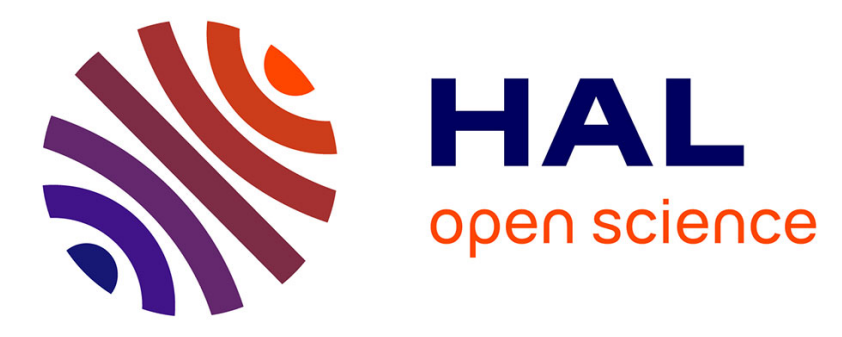

\title{
La dysarthrie au cours de la maladie de Parkinson. Histoire naturelle de ses composantes : dysphonie, dysprosodie et dysarthrie
}

S. Pinto, Alain Ghio, Bernard Teston, François Viallet

\section{To cite this version:}

S. Pinto, Alain Ghio, Bernard Teston, François Viallet. La dysarthrie au cours de la maladie de Parkinson. Histoire naturelle de ses composantes : dysphonie, dysprosodie et dysarthrie. Revue Neurologique, 2010, 166 (10), pp.800 - 810. 10.1016/j.neurol.2010.07.005 . hal-01616010

\author{
HAL Id: hal-01616010 \\ https://hal.science/hal-01616010
}

Submitted on 20 Apr 2018

HAL is a multi-disciplinary open access archive for the deposit and dissemination of scientific research documents, whether they are published or not. The documents may come from teaching and research institutions in France or abroad, or from public or private research centers.
L'archive ouverte pluridisciplinaire HAL, est destinée au dépôt et à la diffusion de documents scientifiques de niveau recherche, publiés ou non, émanant des établissements d'enseignement et de recherche français ou étrangers, des laboratoires publics ou privés. 
Disponible en ligne sur

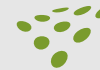

www.sciencedirect.com
Elsevier Masson France

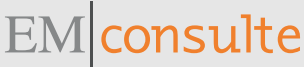

www.em-consulte.com

Journées internationales de la SFN 2010

\title{
La dysarthrie au cours de la maladie de Parkinson. Histoire naturelle de ses composantes : dysphonie, dysprosodie et dysarthrie
}

\section{Dysarthria across Parkinson's disease progression. Natural history of its components: Dysphonia, dysprosody and dysarthria}

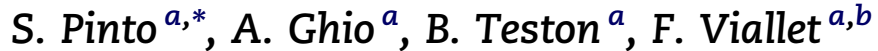 \\ ${ }^{a}$ UMR 6057, laboratoire parole et langage (LPL), CNRS, université Aix-Marseille, BP 80975, 5, avenue Pasteur, 13604 \\ Aix-en-Provence cedex 1, France \\ ${ }^{\mathrm{b}}$ Service de neurologie, centre hospitalier du Pays-d'Aix, Aix-en-Provence, France
}

I N F O A R T I C L E

Historique de l'article :

Disponible sur Internet le

26 août 2010

Mots clés :

Maladie de Parkinson

Parole

Voix

Dysarthrie

Dysphonie

Dysprosodie

Évaluations perceptive et

instrumentale

Keywords:

Parkinson's disease

Speech

Voice

Dysarthria

Dysphonia

Dysprosody

Perceptual and instrumental

assessments

\section{R É S U M É}

La dysarthrie caractérise un trouble de l'exécution motrice de la parole, dont l'origine est une lésion du système nerveux central et/ou périphérique. La dysarthrie hypokinétique de la maladie de Parkinson présente une insuffisance prosodique qui se manifeste par une monotonie d'intensité et de hauteur, une diminution de l'accentuation, un débit variable et une possible imprécision des phonèmes. Dans la plupart des cas, la voix est rauque et soufflée. Ce symptôme peut affecter tant la qualité de la voix et de la parole que l'intelligibilité ou la prosodie, si bien que nombre de patients se plaignent de troubles de la parole perturbant leur communication dans la vie quotidienne. Les évaluations perceptive et instrumentale utilisent de nombreuses méthodes d'investigation permettant de comprendre un peu plus la physiopathologie de la dysarthrie et d'adapter ainsi les traitements ; en effet, la dopathérapie, la neurochirurgie fonctionnelle et la prise en charge orthophonique sont des traitements d'efficacité variable sur la qualité de la voix et de la parole.

C 2010 Elsevier Masson SAS. Tous droits réservés.

\begin{abstract}
A B S T R A C T
Dysarthria refers to a collective name for a group of neurologic motor speech disorders, resulting from central and/or peripheral nervous system abnormalities. Speech alteration in Parkinson's disease, so-called hypokinetic dysarthria, presents with prosodic insufficiency, related to a monotony of pitch and intensity, a reduction of accentuation, variable speech rate and possible phoneme imprecision. In most cases, voice is harsh and breathy. This symptom can affect both voice and speech quality, as well as prosody and intelligibility. As a consequence, many patients complain about speech impairments, which affect their
\end{abstract}

\footnotetext{
* Auteur correspondant.

Adresse e-mail : serge.pinto@lpl-aix.fr (S. Pinto).

0035-3787/\$ - see front matter (C) 2010 Elsevier Masson SAS. Tous droits réservés.
} doi:10.1016/j.neurol.2010.07.005 
communication in daily living activities. Perceptual and instrumental assessments require different and numerous investigation methods, which use may help to further understand the specific dysarthria pathophysiology. This is of importance in order to adjust treatments for dysarthria; as a matter of fact, dopatherapy, functional neurosurgery or even behavioural speech therapy have variable effects on voice and speech quality in Parkinson's disease.

(c) 2010 Elsevier Masson SAS. All rights reserved.

\section{Définition de la dysarthrie}

L'expression anglo-saxonne motor speech disorders fait référence à un ensemble de signes affectant la planification, la programmation, le contrôle et/ou la production de la parole. Les troubles moteurs de la parole ainsi définis ne résultent donc pas de déficits structuraux des organes de la parole ou de troubles de l'audition. On les distingue des troubles du langage (le groupe des aphasies), que l'on associera alors plus volontiers à l'altération de processus cognitifs de plus haut niveau. Cette terminologie est principalement basée sur une approche qui distingue ensuite les troubles moteurs de la parole en deux expressions : le groupe des dysarthries et l'apraxie de la parole.

Dysarthria is a collective name for a group of neurologic speech disorders resulting from abnormalities in the strength, speed, range, steadiness, tone, or accuracy of movements required for control of the respiratory, phonatory, resonatory, articulatory, and prosodic aspects of speech production. The responsible pathophysiologic disturbances are due to central or peripheral nervous system abnormalities and most often reflect weakness; spasticity; incoordination; involuntary movements; or excessive, reduced, or variable muscle tone (Duffy, 2005, page 5). Cette définition de la dysarthrie est inspirée des travaux de Darley et al. (1969a, 1969b, 1975). Elle caractérise un trouble de l'exécution motrice de la parole, dont l'origine est une lésion du système nerveux central ou périphérique ; elle envisage de multiples altérations possibles lors de la réalisation motrice de la parole, pouvant influencer la respiration, la phonation, l'articulation, la résonance et/ou la prosodie.

\section{Historique du terme " dysarthrie "}

Duffy (2007) a récemment rapporté quelques éléments historiques rappelant que le terme dysarthrie ne renvoie effectivement pas qu'à des troubles arthriques. Ce fut le cas pendant longtemps, mais plusieurs auteurs (Liepmann, 1913 ; Head, 1926 ; cités dans Duffy, 2007) ont regroupé, au début du $\mathrm{XX}^{\mathrm{e}}$ siècle, les conséquences des lésions du système sensorimoteur de la parole sous les termes de dysarthrie (troubles de l'articulation) ou anarthrie (absence d'articulation). Cette définition dissociait clairement la dysarthrie de l'aphémie décrite par Broca (1861), maintenant appelée aphasie motrice de Broca. Il est alors apparu comme une évidence de faire correspondre l'association spécifique de signes définissant un trouble moteur de la parole particulier à une topologie bien précise de lésion cérébrale. Plusieurs tentatives de classification des dysarthries se sont alors engagées, bénéficiant et se heurtant à la fois à des approches multi- et pluridisciplinaires : il est en effet difficile de synthétiser en une classification simple et exhaustive des données neuroanatomiques, étiolo- giques, sémiologiques et perceptives. Les travaux de la première partie du $\mathrm{XX}^{\mathrm{e}}$ siècle ont toutefois reconnu que les caractéristiques perceptives de chaque dysarthrie dépendaient plus de la localisation de la lésion neurologique, substrat anatomique de la pathologie associée, que de son étiologie (Luchsinger et Arnold, 1965, cité dans Duffy, 2007).

Les travaux émanant de la seconde moitié du $\mathrm{XX}^{\mathrm{e}}$ siècle se sont alors attelés à inclure sous le terme générique de dysarthrie l'ensemble des altérations pouvant y être associées. Les travaux de Darley et al. (1969a, 1969b, 1975) ont fortement contribué à ne pas dissocier troubles supralaryngés (articulatoires) et laryngés : les troubles respiratoires, phonatoires, de la résonance et prosodiques ont alors été inclus dans la définition. Dans un tel contexte, les utilisations multiples et souvent différentes du terme dysarthrie peuvent prêter à confusion et il reste important de se rappeler que la dysarthrie demeure un signe faisant partie d'un tableau clinique plus large, relevant de la symptomatologie globale d'une pathologie dite du mouvement ; elle est par nature associée à une lésion du système nerveux central ou périphérique. Afin d'être le plus descriptif possible, il conviendrait peut-être de parler de troubles articulatoires et/ou phonatoires pouvant être d'origine neurologique ou non. La notion de trouble arthrique correspondrait alors à un dysfonctionnement de l'articulation supralaryngée, d'origine neurologique (e.g. maladie neurodégénérative) ou non (e.g. chirurgie ablative de la langue). La dysphonie serait définie comme un trouble pneumophonatoire d'origine neurologique, morphologique ou dysfonctionnel, c'est-à-dire incluant toutes les formes de dysfonctionnement laryngé quelle que soit son origine (Fig. 1). C'est un débat qui mériterait un consensus pluridisciplinaire afin de clarifier certains aspects de cette terminologie ; de fait, le terme " dysarthrophonie » apparaît fréquemment afin de rendre compte à la fois des troubles articulatoires et phonatoires liés à la dysarthrie parkinsonienne (Ackermann et Ziegler, 1999 ; Klostermann et al., 2008) ou encore le terme de " dysarthropneumophonie " (Moreau et al., 2010). Le terme " neurodysarthrophonie " serait peut-être celui qui définirait le plus précisément le concept actuel de la dysarthrie, c'est-àdire un dysfonctionnement arthrique (articulatoire) et/ou phonatoire d'origine neurologique (Fig. 1).

L'orthogonalité terminologique dysarthrie/dysphonie peut s'avérer problématique, car si le terme de dysphonie est clairement identifié comme un trouble de la phonation, la dysarthrie est souvent assimilée à tort à un trouble arthrique exclusif, analogie erronée dans la mesure où des troubles articulatoires issus, par exemple, d'une chirurgie ablative orofaciale ne rentrent pas dans le champ de la dysarthrie. Inversement, une hypophonie parkinsonienne, c'est-à-dire une forme de dysphonie, entre dans le champ de la dysarthrie car il s'agit d'un dysfonctionnement d'origine neurologique. 


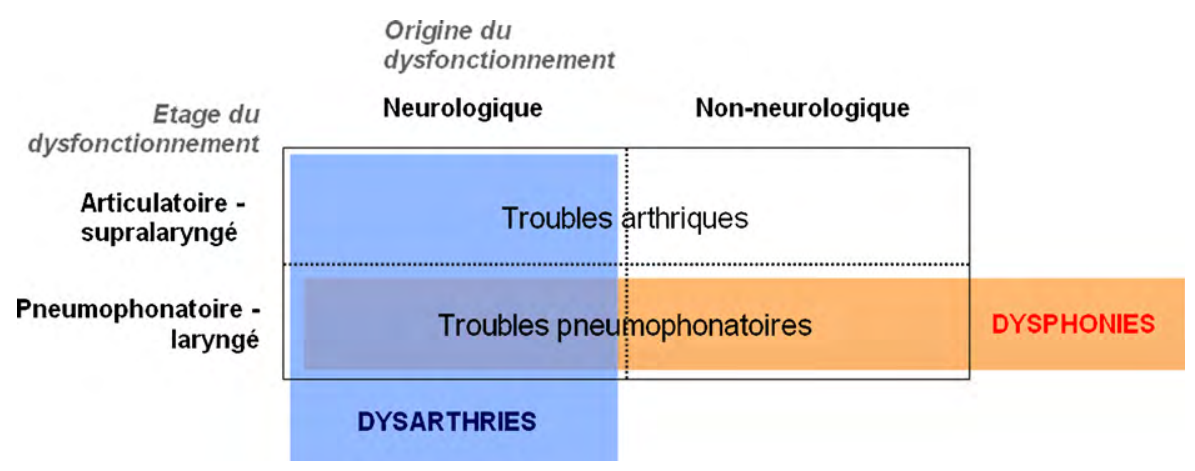

Fig. 1 - Dysarthrie et dysphonie : terminologies et réalités fonctionnelles. Dysarthria and dysphonie: terminologies and functional realities.

Remacle (1999) définit d'ailleurs ce trouble comme une "dysphonie dysarthrique ». Cette problématique se répercute d'ailleurs dans la prise en charge clinique à l'intersection des concepts, c'est-à-dire pour les troubles pneumophonatoires d'origine neurologique. En effet, les patients atteints de ces troubles sont à la croisée de la prise en charge neurologique, du fait de leur maladie, mais aussi de l'ORL, généralement en charge des dysphoniques. De la même manière, il convient de ne pas ignorer la dimension prosodique dans la production de parole, celle-ci concernant les aspects bien spécifiques de hauteur et d'intensité avec leurs modulations respectives, tout autant que le rythme et les dimensions de débit et de durée faisant référence à la segmentation du contenu linguistique.

\section{Maladie de Parkinson et troubles de la production de parole}

La maladie de Parkinson (MP) représente un modèle particulier de dysfonctionnement du système nerveux central (SNC), caractérisé par une dénervation dopaminergique nigro-striatale progressive : il en résulte un dysfonctionnement chronique du système des noyaux gris centraux, dont le rôle est essentiel dans le contrôle de l'exécution des plans moteurs appris. Parmi les activités motrices, la production de parole met particulièrement en évidence les notions d'automatisation (après apprentissage et acquisition) et d'organisation séquentielle ou simultanée des plans moteurs. La production de parole est un système dynamique dont le comportement à un moment donné dépend de ses états antérieurs. Cette spécificité permet d'introduire la notion de " geste articulatoire ", dont l'organisation complexe est sous le contrôle du SNC et notamment des noyaux gris centraux (Viallet et Gayraud, 2005). Les troubles de la production de parole au cours de la MP constituent un des marqueurs cliniques représentatifs de l'évolution du handicap moteur et cognitif ; à l'instar d'autres activités motrices complexes comme les troubles de l'équilibre et de la marche, les troubles de la parole, qui pénalisent particulièrement l'autonomie des patients en détériorant leur capacité de communication au cours de l'évolution de la maladie, voient leur correction par le traitement médicamenteux devenir de plus en plus problématique.
Dans sa pratique clinique au quotidien, le neurologue ne dispose actuellement que d'un outil rudimentaire d'évaluation des troubles de la parole chez les patients parkinsoniens ; il s'agit de l'item 18 (parole) de l'échelle Unified Parkinson's Disease Rating Scale (UPDRS ; Fahn et al., 1987) qui décrit cinq stades de sévérité croissante :

- 0 = parole normale ;

- 1 = baisse légère de l'intonation et du volume ;

- 2 = parole monotone, brouillée mais compréhensible, nettement perturbée ;

- 3=perturbation marquée de la parole, difficile à comprendre ;

- 4 = parole inintelligible.

Au-delà de l'item UPDRS qui reste un outil séméiologique pratique mais sommaire, le neurologue, confronté à la prise en charge des troubles de la parole, se trouve logiquement impliqué dans une démarche pluridisciplinaire dont le but est d'évaluer de façon exhaustive les anomalies des différentes fonctions intervenant dans le système de production de parole (respiration, phonation, résonance et articulation), sans ignorer les dimensions cognitives et psychologiques de la communication parlée. L'évaluation des troubles de la parole dans la MP reste basée pour l'essentiel sur l'analyse perceptive, laquelle permet d'appréhender l'ensemble des fonctions impliquées dans le système de production de parole, les différentes méthodes instrumentales étant par nature limitées dans leur champ d'analyse à l'une de ces fonctions, exception faite toutefois pour les méthodes multiparamétriques d'analyse acoustique et aérodynamique dont l'exhaustivité par rapport à la complexité du signal de parole dépend aussi des applications disponibles.

\section{Histoire naturelle de la dysarthrie au cours de la maladie de Parkinson}

Dans une étude rétrospective de confrontation anatomoclinique, le délai moyen de survenue de la dysarthrie, notifiée dans l'observation clinique, a été retrouvé à 84 mois après le début de la MP (Müller et al., 2001), ce qui illustre la prise en compte insuffisante des troubles de la production de parole qui apparaissent en fait dès le début de la MP et même au cours 
de la période présymptomatique comme cela a été démontré dans une étude longitudinale sur des enregistrements analysés de façon rétrospective chez un patient atteint de MP (Harel et al., 2004). Dans une enquête épidémiologique suédoise (Hartelius et Svensson, 1994), portant sur 250 patients parkinsoniens ayant répondu à l'envoi d'un questionnaire, $70 \%$ se considéraient comme souffrant d'altérations de la voix et de la parole en rapport avec leur MP : la voix était fréquemment qualifiée comme faible, rauque et/ou monotone, et la parole comme trop lente avec articulation imprécise et démarrage difficile. Il en résultait l'expérience de difficultés à se faire entendre au sein d'un groupe ou dans une ambiance bruyante $(60 \%)$ et à se faire comprendre par des personnes familières (37\%) et non familières ( $42 \%)$. En dépit de cette gêne perçue, alors que $46 \%$ des patients bénéficiaient d'une kinésithérapie, seuls 2 à $3 \%$ avaient eu recours à une prise en charge orthophonique.

L'histoire naturelle des troubles de la production de parole au cours de la MP a été résumée par Selby dès 1968, peu avant le développement des médicaments à action dopaminergique : "Dans la grande majorité des cas de paralysie agitante (maladie de Parkinson), les troubles de la parole deviennent de plus en plus évidents au cours de la progression de la maladie. Les points d'accentuation disparaissent, le volume vocal diminue, tandis que la prononciation des consonnes se dégrade et que la phrase s'achève dans un murmure. Alors qu'au début de la maladie la voix est faible, monotone (sans variation de hauteur), l'aggravation progressive de la dysarthrie conduit à une diction devenant inaudible et inintelligible. Tandis que la lenteur générale des mouvements se traduit aussi dans le débit de parole dans certains cas, d'autres patients parlent vite en emmêlant les mots et quelques-uns réalisent une accélération des mots jusqu'à la fin de la phrase, imitant la festination de la marche " (Selby, 1968).

En dépit de ce polymorphisme séméiologique, les troubles de la parole de la MP restent conventionnellement qualifiés de dysarthrie ; les concepts plus élaborés de dysarthrophonie ou de dysarthropneumophonie, bien que plus adaptés, ne parviennent pas plus à englober l'ensemble des anomalies complexes observées au cours de la MP.

Dans le but de préciser l'évolution des troubles de la parole au cours de la progression de la MP, deux études épidémiologiques transversales ont été conduites. Une première étude (Logeman et al., 1978) a concerné 200 patients parkinsoniens évalués en l'absence de traitement antiparkinsonien dans une tâche de lecture à voix haute et de conversation spontanée. L'analyse perceptive a retrouvé une prédominance nette de la dysphonie (89\% des patients) ; les troubles de l'articulation et du débit étant plus rares (respectivement $45 \%$ et $20 \%$ des patients) de même que les troubles de résonance (10\% des patients), l'absence de toute anomalie ne concernant que $11 \%$ des patients. Ces données ont suggéré une progression " caudorostrale " des troubles du conduit vocal au cours de la maladie, débutant au niveau laryngé pour atteindre ensuite la partie postérieure de la constriction linguopalatine, puis sa partie antérieure et enfin la constriction bilabiale. Une autre étude plus récente (Ho et al., 1998) a été conduite chez 200 patients parkinsoniens évalués avec leur traitement antiparkinsonien habituel dans une tâche de conversation spontanée. L'analyse perceptive a montré l'absence de toute anomalie chez $26 \%$ des patients. Parmi les $74 \%$ montrant des anomalies de sévérité variable, la dysphonie prédominait d'autant plus que l'atteinte globale de la communication parlée était moins sévère (94\% dans le groupe d'atteinte légère, $72 \%$ dans le groupe d'atteinte modérée, $46 \%$ dans le groupe d'atteinte sévère et $14 \%$ dans le groupe d'atteinte extrême). Les troubles de l'articulation et du débit n'apparaissaient qu'à partir du groupe d'atteinte modérée et prenaient progressivement une importance croissante allant de pair avec la sévérité globale des troubles de parole. Ces résultats confirment le caractère précoce de la dysphonie au cours de la MP ; toutefois, la progression de la maladie s'exprime ensuite davantage par les troubles de l'articulation et du débit, lesquels perturbent l'intelligibilité de façon plus nette.

Cette précession de la dysphonie, mais aussi de la dysprosodie, a été rapportée chez des patients parkinsoniens avec handicap moteur faible et indépendants dans leur vie quotidienne, évalués en conversation "spontanée " au cours d'un entretien semi-structuré, les seules anomalies observées étant la réduction de variabilité de la hauteur et l'augmentation du nombre de pauses « non remplies » de durée supérieure à une seconde (Pitcairn et al., 1990). L'image d'une trajectoire évolutive globale des troubles de la parole au cours de la MP ne doit toutefois pas faire oublier la très grande variabilité interindividuelle du phénotype clinique selon la topographie précise de la dénervation dopaminergique et selon les stratégies d'adaptation développées par les patients euxmêmes de façon plus ou moins consciente, ce qui affectera de façon également variable la production de parole (Metter et Hanson, 1986) ; il faut aussi tenir compte de la problématique générale de reproductibilité des observations et des enregistrements chez un patient donné.

\section{Analyse perceptive du trouble de production de parole au cours de la maladie de Parkinson : la dysarthrie hypokinétique}

La contribution de l'équipe de la Mayo Clinic (Darley et al., 1969a, 1969b, 1975), reprenant le principe d'une classification étiologique des dysarthries d'origine neurologique, a été largement reconnue dans la littérature, notamment pour ce qui concerne le concept de dysarthrie hypokinétique attribué à la MP. L'originalité de l'approche résidait dans l'analyse globale du système de production de parole incluant la respiration, la phonation ainsi que la résonance, l'articulation et surtout la prosodie. La méthodologie était basée sur l'analyse perceptive par trois juges des enregistrements d'un texte lu à voix haute par les patients : la cotation comportait 38 paramètres, avec une échelle de 1 (normal) à 7 (perturbation maximale).

Les dix dimensions les plus déviantes (score moyen supérieur ou égal à 1,5), considérées comme caractéristiques de la parole parkinsonienne, ont été établies chez 32 patients et sont dans l'ordre décroissant : la monotonie de hauteur, la réduction de l'accentuation, la monotonie de l'intensité, l'imprécision des consonnes, les pauses inappropriées, les accélérations brèves, la raucité de la voix, la voix « soufflée », la 
hauteur moyenne et le débit variable. Pour la hauteur moyenne, la déviation, bien que peu marquée, était orientée vers l'abaissement, tandis que le débit moyen était plutôt orienté vers l'accélération. Le regroupement de certaines de ces perturbations (monotonie de hauteur et d'intensité, réduction d'accentuation, accélérations brèves, débit variable et imprécision des consonnes) a conduit à mettre en exergue le " cluster " principal d'insuffisance prosodique comme caractéristique de la dysarthrie hypokinétique. Accessoirement, les perturbations de la voix y ajoutaient le « cluster » secondaire d'incompétence phonatoire. Avec le temps et malgré des limites dont la quasi-absence de reproductibilité n'est pas la moindre, cette description de Darley et al. (1975) reste très en faveur dans la pratique clinique (Kent et al., 2000), au moins pour ce qui concerne la dysarthrie hypokinétique. L'évaluation auditive-perceptive demeure un outil clinique important et adéquat, dans la mesure où elle permet de décrire les multiples dimensions de la parole dysarthrique : une étude de congruence entre différents juges d'écoute utilisant les critères de la Mayo Clinic renforce cet argument (Bunton et al., 2007). Il convient toutefois de commenter sa validité dans le contexte précis de la MP en considérant notamment des facteurs pouvant influencer la performance des patients tels que la situation de production de parole et surtout les traitements antiparkinsoniens.

L'influence de la situation de production de parole sur l'intelligibilité est une notion décrite dans la parole normale : une diminution de l'intelligibilité est observée dans la parole spontanée en comparaison avec la parole lue (Meunier et Floccia, 1999). Cela a été spécifiquement analysé chez un patient parkinsonien en comparant notamment, sur des corpus de matériel verbal identiques, la parole spontanée et la lecture à voix haute (de phrases transcrites à partir du discours spontané) : les résultats ont montré une altération beaucoup plus sévère de l'intelligibilité pour la parole spontanée (70 \%) que pour la parole lue (20\%), cette altération étant due pour l'essentiel à un nombre beaucoup plus élevé de dysfluences (avec dégradation de la structure des spectrogrammes acoustiques) en parole spontanée (Kempler et Van Lancker, 2002). Dans cette étude, il n'était pas retrouvé de différence significative sur l'intensité du signal de parole entre les deux situations : toutefois, il est de constatation courante en pratique clinique de percevoir une intensité plus faible en parole spontanée qu'en parole lue. L'atténuation des troubles en parole lue serait liée à l'utilisation par le patient du modèle " externe " apporté par le texte écrit lui permettant de compenser la dégradation du modèle « interne » des " gestes articulatoires " résultant du dysfonctionnement du contrôle exercé par le système des ganglions de la base. La MP comporte un déficit du traitement de l'information sensorielle (et particulièrement des informations d'origine proprioceptive) destinée à la calibration fine des activités motrices par l'intermédiaire de " modèles internes » utilisables pour la réalisation des mouvements (Kent et al., 2000) : cet aspect a été souligné pour la sphère orofaciale, mais concerne aussi le conduit vocal, la sphère laryngée et la musculature respiratoire. Cette interprétation se prête notamment à l'explication de la réduction du volume vocal observée chez les patients parkinsoniens en dépit de la perception par ces mêmes patients d'un volume normal (Ho et al., 1999) et se trouve à la base des principes de la méthode LSVT pour la prise en charge rééducative de la dysarthrie hypokinétique (Ramig et al., 1994). Cette même interprétation peut être étendue aux aspects temporels de la production de parole en considérant aussi le déficit de l'estimation du temps et de la discrimination temporelle dans la MP : outre l'impact lié au tremblement, qui peut, au titre d'activité rythmique agissant comme " modèle interne ", influencer le décours temporel de la production de parole avec des effets d'attraction, les troubles kinesthésiques, compte tenu des contraintes biomécaniques inhérentes à la physiologie du conduit vocal et des articulateurs, peuvent être incriminés dans la genèse des anomalies concernant les phénomènes de durée (durée des occlusions ou des constrictions, durée des voyelles et des noyaux syllabiques, rythmes alternatifs) qui affectent tout particulièrement les réalisations prosodiques et articulatoires et par conséquent l'intelligibilité (Kent et al., 2000).

\section{Apports de l'évaluation acoustique et aérodynamique à la description des composantes de la dysarthrie parkinsonienne}

\subsection{Dysphonie parkinsonienne}

Elle correspond aux anomalies de fonctionnement du vibrateur laryngé concernant la hauteur, l'intensité et le timbre, lesquelles ont été rapportées de façon détaillée et exhaustive (Robert et Spezza, 2005). En ce qui concerne la hauteur, les analyses perceptives et les mesures acoustiques retrouvent des résultats contrastés ; toutefois, la majorité des études rapporte une élévation de la fréquence fondamentale (F0) moyenne chez les patients parkinsoniens au cours de la voyelle tenue, de la lecture d'un texte ou en parole spontanée. Il y a cependant des biais tenant à l'âge (dont on connaît l'effet d'élévation de la F0), au sexe (effets plus marqués pour le sexe masculin), à la durée d'évolution de la maladie (avec la mise en jeu de stratégies compensatrices), aux effets des traitements antiparkinsoniens (notamment de la L-dopa, les études étant plus rares sur les patients en période " off ") et enfin à la variabilité de performance interindividuelle et intra-individuelle, sans parler de l'hétérogénéité des méthodes d'évaluation ou de mesure. Il paraît donc plus réaliste d'envisager une grande diversité des anomalies de la F0 moyenne qui peut être : soit abaissée comme conséquence initiale du déficit en dopamine responsable d'une réduction de la pression sous-glottique et d'une incompétence phonatoire par hypokinésie de la musculature laryngée ; soit élevée sous l'effet correcteur du traitement antiparkinsonien et sous l'effet de stratégies de compensation visant à optimiser la fermeture laryngée ; soit inchangée par équilibre entre les facteurs d'élévation et d'abaissement (Viallet et Teston, 2007). En ce qui concerne l'effet sexe, la modification sexe-dépendante de la F0 rapportée pour une population de patients dysphoniques d'étiologies diverses (augmentation pour les hommes, diminution pour les femmes ; Yu et al., 2007) est une notion à prendre également en considération pour l'interprétation de l'ensemble de ces données.

En ce qui concerne l'intensité, les analyses perceptives et les mesures acoustiques retrouvent également des résultats contrastés avec une dominante toutefois pour une faible 
réduction de l'intensité moyenne ; les mêmes commentaires que pour la hauteur peuvent être formulés concernant notamment les effets de la tâche utilisée (voyelle tenue, lecture de texte ou parole spontanée). L'incompétence phonatoire avec la réduction de la pression sous-glottique est un facteur évident de la réduction de l'intensité moyenne (Viallet et Teston, 2007). Les anomalies du timbre (soufflé ou éraillé), ainsi que le caractère tremblé de la voix, ont été retrouvées de façon constante dans les études perceptives.

Les mesures acoustiques au cours de la voyelle tenue ont permis d'objectiver ces anomalies du timbre en mettant en évidence une augmentation des variations cycle à cycle de la F0 (jitter) et de l'intensité (shimmer), ainsi que des variations à plus long terme dues notamment au tremblement, avec une réduction du rapport signal-bruit. Les études en laryngoscopie ont apporté un éclairage direct sur les anomalies du larynx dans la MP en mettant en évidence une béance glottique par défaut d'accolement cordal, parfois une hypertonie des bandes ventriculaires, et un tremblement, soit au niveau du plan cordal, soit au niveau du tractus vocal sus-glottique (Jiang et al., 1999 ; Yuceturk et al., 2002).

\subsection{Dysprosodie parkinsonienne}

L'insuffisance prosodique constitue probablement la marque la plus spécifique des troubles de la parole dans la MP : selon la description de la Mayo Clinic (Darley et al., 1975), six des dix dimensions les plus déviantes relèvent de la dysprosodie (monotonie de hauteur et d'intensité, réduction d'accentuation, pauses inappropriées, accélérations brèves et débit variable). La monotonie de hauteur se traduit par une réduction de la dynamique de fréquence et donc de la gamme tonale, ce qui altère la mélodie avec réduction des contrastes de hauteur entre les points cibles dont la structuration reste globalement préservée (Teston et Viallet, 2005). L'étude de la distribution de la F0 retrouve, notamment chez les parkinsoniens en période " off ", une perte de la partie haute de la gamme tonale (Viallet et al., 2000). La monotonie de l'intensité, souvent rapportée dans les analyses perceptives, est plus difficile à objectiver avec les analyses acoustiques instrumentales.

En ce qui concerne le rythme (c'est-à-dire le débit avec la durée et les pauses), la festination de la parole, la palilalie et les pseudo-bégaiements avec les dysfluences sont des anomalies décrites depuis longtemps dans la MP et remises en exergue récemment (Monfrais-Pfauwadel, 2005). L'organisation temporelle de la parole dans la MP a été abordée par l'analyse fine du signal acoustique sur des extraits de parole lue avec écoute auditive attentive (Duez, 2005) : la vitesse de parole a tendance à être plus lente dans la MP et elle semble corrélée à un temps de pause plus élevé ; la durée moyenne des pauses a été retrouvée significativement plus longue chez dix patients parkinsoniens en période " off " par rapport à dix sujets contrôles d'âge moyen équivalent, alors que la durée moyenne des suites sonores ne montrait pas de différence significative entre parkinsoniens et sujets contrôles ; de plus, chez les patients parkinsoniens, des pauses étaient observées à l'intérieur de mots, ce qui n'était pas le cas chez les sujets contrôles, tandis que des pauses à l'intérieur de syntagmes étaient très majoritairement présentes chez les parkinsoniens, ces aspects entraînant une différence significative dans la distribution des pauses entre les deux groupes; enfin, de nombreuses dysfluences, telles que les omissions, les faux départs et les répétitions, apparaissaient de façon quasiexclusive chez les parkinsoniens. Ces données, en accord avec certaines études antérieures (Metter et Hanson, 1986 ; Hammen et Yorkston, 1996), suggèrent que l'allongement anormal des pauses, les pauses à l'intérieur de mots et de syntagmes et les dysfluences créent une rupture dans la cohésion des unités linguistiques, ce qui perturbe l'attente perceptive des auditeurs et dégrade l'intelligibilité (Duez, 2005). Les consonnes occlusives et fricatives non voisées sont réduites de manière significative dans la parole parkinsonienne, alors que les voyelles sont légèrement plus longues par rapport à la parole normale; la plupart des patients tendent à maintenir les contrastes de durée des consonnes et des voyelles, ce qui suggère que les contraintes de bas niveau opèrent de manière similaire et avec le même poids dans la MP (Duez, 2009). Par ailleurs, l'allongement final est conservé chez les patients : la fonction syntaxique de la prosodie est conservée chez les patients parkinsoniens, du moins aux stades précoce et moyen de la maladie (Duez et al., 2009).

\subsection{Troubles articulatoires dans la maladie de Parkinson}

Outre l'imprécision des consonnes, déviance assez peu spécifique puisqu'elle est retrouvée dans toutes les dysarthries selon les données de la Mayo Clinic (Darley et al., 1975), l'évaluation perceptive révèle que les anomalies articulatoires portent essentiellement sur les consonnes occlusives dans la MP, lesquelles sont perçues comme des constrictives, témoignant d'une fermeture insuffisante du conduit vocal (Robert et Spezza, 2005). Les analyses acoustiques retrouvent de ce fait, à la place d'un silence dû à une occlusion normalement réalisée, un signal correspondant à un bruit de friction de faible intensité dû au passage d'air, ce qui est défini comme le phénomène de spirantisation (Kent et Rosenbek, 1992), l'intensité de ce bruit de friction étant corrélée avec le degré de dysarthrie chez les patients parkinsoniens. Il semble exister chez certains patients une tendance rhinolalique, mais globalement il n'est pas retrouvé de différence significative entre groupes de parkinsoniens et de sujets contrôles. Pour ce qui concerne les voyelles, la tendance à la réduction de la surface du triangle vocalique rapportée chez les patients peut rendre compte de la dédifférenciation des voyelles, ce qui contribue avec les phénomènes de spirantisation et de coarticulation (Tjaden, 2000) à la réduction des contrastes acoustiques dans la parole parkinsonienne. Les mesures aérodynamiques (débit d'air oral, pression intra-orale) au cours de la réalisation de consonnes occlusives ont toutefois retrouvé une préservation de la hiérarchie de l'opposition de voisement chez les parkinsoniens avec des pics de pression restant plus élevés pour les consonnes non voisées que pour les consonnes voisées (Locco, 2004).

\section{Effets des traitements}

\subsection{Dopathérapie}

L'influence des traitements antiparkinsoniens, notamment de la L-dopa, sur la performance motrice des patients, est une 
évidence clinique : la pratique au quotidien suggère que les troubles de la production de parole, à l'instar des troubles moteurs en général, sont atténués par le traitement, au moins au début de la maladie lorsque la dysphonie et la dysprosodie prédominent ; avec la progression de la maladie en effet, les troubles articulatoires se développent et l'ensemble des perturbations, comme les signes « axiaux » en général, devient de moins en moins réversible sous l'effet des traitements. Dans une étude de phrases lues chez dix patients parkinsoniens de sexe masculin (âge moyen : 71 ans ; durée moyenne de maladie : 11 ans), aucun effet significatif de la L-dopa n'était retrouvé sur la durée des voyelles et la surface du triangle vocalique (Poluha et al., 1998). L'étude de la production de voyelle tenue chez 15 patients parkinsoniens (dix hommes et cinq femmes, âge moyen : 67 ans ; durée moyenne de maladie : quatre ans) n'a montré aucun effet significatif de la L-dopa sur la hauteur moyenne (F0), mais une augmentation faible mais significative de l'intensité moyenne et une tendance à l'augmentation du débit d'air (Jiang et al., 1999) ; en ce qui concerne la hauteur moyenne, l'analyse individuelle montre que la L-dopa l'augmente chez $50 \%$ des patients et la réduit chez $50 \%$ des patients. Dans une autre étude de la production de voyelle tenue chez 20 patients parkinsoniens (dix hommes et dix femmes, âge moyen : 63 ans ; durée moyenne de maladie : sept ans), la L-dopa a entraîné une augmentation significative de la hauteur moyenne (F0) et du rapport signal-bruit, ainsi qu'une réduction significative du jitter et du tremblement (Sanabria et al., 2001) : l'analyse individuelle montre que la L-dopa élève la F0 chez 17 patients et la réduit chez les trois autres patients. Enfin, une étude de la production de voyelle tenue et de la lecture d'un texte chez neuf patients parkinsoniens (six hommes et trois femmes, âge moyen : 69 ans ; durée moyenne de maladie : 11 ans) n'a montré aucun effet significatif de la L-dopa sur la F0 moyenne (Goberman et al., 2002) ; l'analyse individuelle retrouve toutefois une augmentation de la F0 sous l'effet de la L-dopa chez six patients pour la voyelle tenue. Il apparaît donc des résultats controversés, à savoir, soit l'absence d'effet de la L-dopa, soit une augmentation de la F0: cela illustre la nécessité d'optimiser le contrôle pharmacologique de l'effet clinique de la L-dopa en vérifiant l'amélioration du score moteur global chez les patients, de tenir compte de la durée de la maladie et enfin d'accepter une hétérogénéité de comportement dans l'adaptation au trouble de production de parole chez les patients, certains mettant en place des stratégies de compensation telles que l'augmentation de constriction laryngée. Dans deux études récentes (Jankowski et al., 2004 ; Viallet et al., 2002), l'effet de la Ldopa apparaît plus clairement avec, d'une part, une augmentation significative de la F0 moyenne et de l'étendue de la gamme tonale au cours de la lecture d'un texte chez dix patients parkinsoniens de sexe masculin (âge moyen : 57ans ; durée moyenne de maladie : dix ans) et, d'autre part, une augmentation significative de la F0 moyenne, de l'intensité moyenne et du débit d'air moyen au cours de la production de la voyelle [a] tenue chez 44 patients parkinsoniens de sexe masculin (âge moyen : 64 ans ; durée moyenne de maladie : neuf ans).

\subsection{Stimulation du noyau subthalamique}

Les évaluations cliniques de la stimulation du noyau subthalamique (NST) sur la dysarthrie montrent des effets moins prononcés que sur les mouvements des membres. L'amélioration perceptive initiale de l'intelligibilité tend à décroître sur le long terme. Amélioration et perte d'intelligibilité peuvent être observées après opération et la persistance, voire l'apparition, de la dysarthrie demeure une des complications courantes. Pour estimer les effets de la stimulation du NST sur la parole, les études menées s'appuient notamment sur la mesure individuelle de différents paramètres de la voix et de la motricité orofaciale.

Après stimulation du NST, l'intensité et la variabilité de la F0 sont modérément améliorées, la parole étant moins monotone (Dromey et al., 2000 ; Gentil et al., 2001). Les patients parkinsoniens réduisent leur temps de pause et augmentent leur temps maximal de phonation après stimulation du NST (Gentil et al., 2003). Les forces des organes articulatoires sont sensibles à la stimulation du NST (Gentil et al., 2003 ; Pinto et al., 2003), une amélioration des forces des lèvres de $74 \%$ et de $66 \%$ pour la langue étant constatée, ainsi que la réduction des temps de réaction. La stimulation du NST permettrait donc une meilleure activité musculaire (Gentil et al., 1999), reflétant une modulation bénéfique des structures neuronales impliquées dans la production de parole. De plus, les mesures aérodynamiques ont permis de retrouver une amélioration de la pression intra-orale, laquelle est une estimation de la pression sous-glottique (Sarr et al., 2009). Néanmoins les résultats de Dromey et al. (2000) montrent des effets limités sur l'intensité et la fréquence vocale, renforçant l'idée que la stimulation du NST peut avoir des effets différents en fonction des paramètres de la parole et/ou de la voix, étudiés de manière isolée. La stimulation du NST en effet peut s'avérer inefficace chez des patients présentant une dysarthrie sévère (Gentil et al., 1999), aggraver une dysarthrie déjà existante ou engendrer une dysarthrie qualifiée de stimulation-dépendante (Pinto et al., 2005).

D'un point de vue perceptif, des études ont montré qu'après stimulation du NST, le niveau d'intelligibilité pouvait s'aggraver (Rousseaux et al., 2004 ; Santens et al., 2003 ; Törnqvist et al., 2005). Le temps de pause est plus long, la voix plus monotone, il y a des difficultés d'articulation et une accélération de la vitesse de parole, ce qui accentue la sensation d'inintelligibilité (Rousseaux et al., 2004). L'intelligibilité ne semble pas associée à une réduction des capacités motrices élémentaires puisque la mobilité des lèvres, de la langue et autres acteurs musculaires impliqués dans la parole est améliorée (Rousseaux et al., 2004). La perte d'intelligibilité pourrait être due à des paramètres de stimulation inadéquats (mauvais placement des électrodes, trop haute fréquence, amplitude de stimulation inadaptée), car en fonction des paramètres de stimulation, une amélioration de certaines composantes de la parole peut être observée sans pour autant avoir un impact global sur la dysarthrie (Törnqvist et al., 2005). Cette influence non bénéfique sur l'intelligibilité est à mettre en relation avec la localisation des électrodes (Aström et al., 2010 ; Tripoliti et al., 2008). Il doit être mentionné ici que l'effet délétère de la stimulation du NST sur la fluence verbale (Brusa et al., 2001 ; De Gaspari et al., 2006) est une notion importante à prendre en compte.

\subsection{Rééducation orthophonique intensive}

Les effets de la plupart des traitements pharmacologiques ou chirurgicaux restent insatisfaisants (Pinto et al., 2004a) car ils 
n'apportent pas d'amélioration significative de la dysarthrie. En revanche, la prise en charge rééducative a longtemps été négligée. Les méthodes traditionnelles sont basées sur l'entraînement vocal : l'accent est mis sur le travail des muscles de la respiration, la phonation, l'articulation supralaryngée et la coordination pneumophonique. Les traitements orthophoniques peuvent dans certains cas améliorer la monotonie et l'intelligibilité de la voix, augmenter la durée de la phonation ou encore l'intensité vocale. Les résultats rapportés par les principales études diffèrent par les méthodes et la composante de la production ciblée (pour revue, Rolland-Monnoury et Özsancak, 2007 ; Ramig et Fox, 2007) : rééducation des aspects prosodiques (Scott et Caird, 1983, 1984) ; rééducation diversifiée avec utilisation de feed-back visuel (Robertson et Thomson, 1984) ; rééducation avec exercices de relaxation et feed-back (Johnson et Pring, 1990) ; rééducation de la compétence laryngée (De Angelis et al., 1997) ; rééducation de la phonation, telle que la Lee Silverman Voice Treatment ou LSVT (Ramig et al., 1994). Bien que tous les aspects de la production de la parole puissent être atteints, les approches antérieures visant les troubles de la voix et de la parole chez les patients parkinsoniens, n'ont eu que des effets inconstants et une persistance minime à long terme du bénéfice thérapeutique.

Le développement de la LSVT a permis de démontrer son efficacité à la fois à court et long termes (Ramig et al., 1995, 1996, 2001a, 2001b). Ce programme thérapeutique intensif a pour objectifl'augmentation de l'intensité vocale et l'amélioration de la perception sensorielle de l'effort vocal et de l'intensité. Le patient parle fort et augmente ainsi sa performance respiratoire. L'approche combinée faisant travailler l'adduction des cordes vocales et le contrôle du souffle respiratoire conduirait à un bénéfice persistant sur l'intensité vocale. L'impression perceptive des patients et de leurs proches confirme l'effet positif du traitement dans les situations communicationnelles (Ramig et al., 1996, 2001b). Une étude française (Meteor) consistant à évaluer la méthode sur 62 patients parkinsoniens a rapporté son efficacité (Rolland-Monnoury et Özsancak, 2007). Au-delà de la performance vocale, d'autres effets positifs distribués sur l'ensemble du système de production de parole ont été documentés après traitement LSVT : amélioration de l'articulation (Dromey et al., 1995 ; Sapir et al., 2007), de l'expression faciale (Spielman et al., 2003) ou encore de la déglutition (El Sharkawi et al., 2002).

\section{Modifications d'activations cérébrales associées à la dysarthrie parkinsonienne}

Liotti et al. (2003) ont étudié la réponse hémodynamique cérébrale en tomographie par émission de positons (TEP) lors de diverses tâches de parole, chez cinq patients parkinsoniens. Ces patients ont participé deux fois, avant et après traitement orthophonique intensif issu de la méthode LSVT (Ramig et al., 1994). En plus de mettre en évidence les corrélats anatomofonctionnels de l'efficacité du traitement, ont aussi été révélés avant le traitement une hyperactivation bilatérale des régions corticales motrice/prémotrice, y compris l'aire motrice supplémentaire (AMS), ainsi que du cortex prémoteur latéral inférieur. Une autre étude de ces mêmes auteurs a récemment confirmé ces hyperactivations prétraitement chez dix patients parkinsoniens (Narayana et al., 2010) ; comme l'étude précédente, les données font référence à l'effet de ce traitement orthophonique chez des patients médicamentés. Par ailleurs, la dysarthrie dans la MP a été également associée :

- à une diminution d'activation dans le cortex moteur primaire orofacial droit et les hémisphères cérébelleux bilatéraux ;

- à une augmentation anormale de débit sanguin cérébral régional (DSCr) dans le cortex prémoteur supérieur droit et le cortex préfrontal dorsolatéral (DLPFC) bilatéral ;

- à une hyperactivation de l'AMS (Pinto et al., 2004b).

La dysarthrie parkinsonienne pourrait donc résulter d'un recrutement altéré des principales régions cérébrales motrices (cortex moteur orofacial, cervelet) et d'une plus grande participation des cortex prémoteur et préfrontaux (DLPFC, AMS, cortex prémoteur supérieur). Une étude TEP récente portant sur un sujet a également rapporté l'hyperactivation de l'AMS, associée à une hyperactivation du cortex moteur primaire (Narayana et al., 2009). Des modifications d'activations cérébrales, telles que le recrutement supplémentaire de régions temporales lors de la phonation et de la répétition de phonème, semblent représenter une réorganisation importante sous-tendant la parole parkinsonienne (Sachin et al., 2008). Une autre étude en IRMf a rapporté, chez neuf patients parkinsoniens en condition on-médicaments, une augmentation de DSCr dans le cortex sensorimoteur orofacial droit par rapport à des sujets contrôles (Rektorova et al., 2007). Cette modification d'activation cérébrale a été interprétée par les auteurs comme un phénomène de compensation pour pallier les activations cérébrales réduites, afin de préserver la production de parole chez les patients.

Les patients souffrant de la MP peuvent également présenter des difficultés de langage de plus haut-niveau (Berg et al., 2003). La littérature rapporte des déficits significatifs dans la génération de verbe (Bertella et al., 2002 ; Castner et al., 2008 ; Péran et al., 2003), certains aspects des mécanismes de l'inhibition lexico-sémantique (Castner et al., 2007a), du priming sémantique et affectif (Castner et al., 2007b), ainsi que dans la compréhension (Angwin et al., 2005, 2006, 2007) et le traitement (Grossman et al., 2002) de phrase. De telles difficultés se réfèrent au dysfonctionnement des circuits nonmoteurs des ganglions de la base (Castner et al., 2007a, 2007b). Notamment, l'altération d'un réseau frontotemporal lié à la production de mots isolés a été suggéré (Castner et al., 2008), ainsi que l'hyperactivation des aires pariétales, frontale inférieure droite et temporale postérolatérale gauche lors de la compréhension de phrase (Grossman et al., 2003).

\section{Conflit d'intérêt}

Les auteurs ne déclarent aucun conflit d'intérêt.

\section{Remerciements}

Association France Parkinson, Fondation Fyssen et PHRC 1997 et 2003. 


\section{RÉ FÉ R E N C E S}

Ackermann H, Ziegler W. Dysarthrophonia of Parkinson syndrome. Fortschr Neurol Psychiatr 1999;57:149-60.

Angwin AJ, Chenery HJ, Copland DA, Murdoch BE, Silburn PA. Summation of semantic priming and complex sentence comprehension in Parkinson's disease. Brain Res Cogn Brain Res 2005;25:78-89.

Angwin AJ, Copland DA, Chenery HJ, Murdoch BE, Silburn PA. The influence of dopamine on semantic activation in Parkinson's disease: evidence from a multipriming task. Neuropsychology 2006;20:299-306.

Angwin AJ, Chenery HJ, Copland DA, Murdoch BE, Silburn PA. The speed of lexical activation is altered in Parkinson's disease. J Clin Exp Neuropsychol 2007;29:73-85.

Aström M, Tripoliti E, Hariz MI, Zrinzo LU, Martinez-Torres I, Limousin P, et al. Patient-specific model-based investigation of speech intelligibility and movement during deep brain stimulation. Stereotact Funct Neurosurg 2010;88:224-33.

Berg E, Björnram C, Hartelius L, Laakso K, Johnels B. High-level language difficulties in Parkinson's disease. Clin Linguist Phon 2003;17:63-80.

Bertella L, Albani G, Greco E, Priano L, Mauro A, Marchi S, et al. Noun-verb dissociation in Parkinson's disease. Brain Cogn 2002;48:277-80.

Broca P. Remarques sur le siège de la faculté du langage articulé suivie d'une observation d'aphémie. Bull Soc Anat Paris 1861;36:330-57.

Brusa L, Pierantozzi M, Peppe A, Attibrandi MG, Giacomini P, Mazzone $P$, et al. Deep brain stimulation attentional effects parallel those of 1-dopa treatment. J Neural Transm 2001;108:1021-7.

Bunton K, Kent RD, Duffy JR, Rosenbek JC, Kent JF. Listener agreement for auditory-perceptual ratings of dysarthria. J Speech Lang Hear Res 2007;50:1481-95.

Castner JE, Copland DA, Silburn PA, Coyne TJ, Sinclair F, Chenery HJ. Lexical-semantic inhibitory mechanisms in Parkinson's disease as a function of subthalamic stimulation. Neuropsychologia 2007;45:3167-77.

Castner JE, Chenery HJ, Copland DA, Coyne TJ, Sinclair F, Silburn PA. Semantic and affective priming as a function of stimulation of the subthalamic nucleus in Parkinson's disease. Brain 2007;130:1395-407.

Castner JE, Chenery HJ, Silburn PA, Smith ER, Coyne TJ, Sinclair $\mathrm{F}$, et al. The effects of subthalamic deep brain stimulation on noun/verb generation and selection from competing alternatives in Parkinson's disease. J Neurol Neurosurg Psychiatry 2008;79:700-5.

Darley FL, Aronson AE, Brown JR. Differential diagnostic patterns of dysarthria. J Speech Hear Res 1969;12:246-69.

Darley FL, Aronson AE, Brown JR. Clusters of deviant speech dimensions in the dysarthrias. J Speech Hear Res 1969;12:462-96.

Darley FL, Aronson AE, Brown JR. Hypokinetic dysarthria: disorders of extrapyramidal system. In: Motor speech disorders. Philadelphia: W.B. Saunders; 1975. p. 171-197.

De Angelis EC, Mourão LF, Ferraz HB, Behlau MS, Pontes PAL, Andrade LAF. Effect of voice rehabilitation on oral communication of Parkinson's disease patients. Acta Neurol Scand 1997;96:199-205.

De Gaspari D, Siri C, DiGioia M, Antonini A, Isella V, Pizzolato A, et al. Clinical correlates and cognitive underpinnings of verbal fluency impairment after chronic subthalamic stimulation in Parkinson's disease. Parkinsonism Relat Disord 2006;12:289-95.

Dromey C, Ramig LO, Johnson AB. Phonatory and articulatory changes associated with increased vocal intensity in
Parkinson disease: a case study. J Speech Hear Res 1995;38:751-64.

Dromey C, Kumar R, Lang AE, Lozano AM. An investigation of the effects of subthalamic nucleus stimulation on acoustic measures of voice. Mov Disord 2000;15:1132-8.

Duez D. Organisation temporelle de la parole et dysarthrie parkinsonienne. In: Özsancak C, Auzou P, editors. Les troubles de la parole et de la déglutition dans la maladie de Parkinson. Marseille: Solal; 2005. p. 195-211.

Duez D. Segmental duration in parkinsonian French speech. Folia Phoniatr Logop 2009;61:239-46.

Duez D, Legou T, Viallet F. Final lengthening in parkinsonian French speech: effects of position in phrase on the duration of CV syllables and speech segments. Clin Linguist Phon 2009;23:781-93.

Duffy JR. Motor speech disorders: substrates, differential diagnosis and management. St Louis: Motsby-Yearbook; 2005.

Duffy JR. Motor speech disorders: history, current practice. Future trends and goals. In: Weismer, G, editor. Motor Speech Disorders. San Diego: Plural Publishing; 2007. p. 7-56.

El Sharkawi A, Ramig L, Logemann J, Pauloski B, Rademaker A, Smith C, et al. Swallowing and voice effects of Lee Silverman Voice Treatment: a pilot study. J Neurol Neurosurg Psychiatry 2002;72:31-6.

Fahn S, Elton RL, The committee motUd. Unified Parkinson's Disease Rating Scale. In: Fahn S, Marsden CD, Calne DB, editors. Recent developments in Parkinson's disease. New Jersey: Florham Park, MacMillan, Health Care Information; 1987. p. 153-64.

Gentil M, Garcia-Ruiz P, Pollak P, Benabid AL. Effect of stimulation of the subthalamic nucleus on oral control of patients with parkinsonism. J Neurol Neurosurg Psychiatry 1999;67:329-33.

Gentil M, Chauvin P, Pinto S, Pollak P, Benabid AL. Effect of bilateral stimulation of the subthalamic nucleus on parkinsonian voice. Brain Lang 2001;78:233-40.

Gentil M, Pinto S, Pollak P, Benabid AL. Effect of bilateral stimulation of the subthalamic nucleus on parkinsonian dysarthria. Brain Lang 2003;85:190-6.

Goberman A, Coelho C, Robb M. Phonatory characteristics of Parkinsonian speech before and after morning medication: the ON and OFF states. J Commun Disord 2002;35:217-39.

Grossman M, Lee C, Morris J, Stern MB, Hurtig HI. Assessing resource demands during sentence processing in Parkinson's disease. Brain Lang 2002;80:603-16.

Grossman M, Cooke A, DeVita C, Lee C, Alsop D, Detre J, et al. Grammatical and resource components of sentence processing in Parkinson's disease: An fMRI study. Neurology 2003;60:775-81.

Hammen V, Yorkston K. Speech and pause characteristics following speech rate reduction in hypokinetic dysarthria. J Commun Disord 1996;29:429-45.

Harel B, Cannizzaro M, Snyder PJ. Variability in fundamental frequency during speech in prodromal and incipient Parkinson's disease: a longitudinal case study. Brain Cogn 2004;56:24-9.

Hartelius L, Svensson P. Speech and swallowing symptoms associated with Parkinson's disease and Multiple sclerosis: a survey. Folia Phoniatr Logop 1994;46:9-17.

Head H. Aphasia and kindred disorders of speech. New-York: MacMillan; 1926.

Ho AK, Iansek R, Marigliani C, Bradshaw JL, Gates S. Speech impairment in a large sample of patients with Parkinson's disease. Behav Neurol 1998;11:131-7.

Ho AK, Iansek R, Bradshaw JL. Regulation of parkinsonian speech volume: the effect of interlocuter distance. J Neurol Neurosurg Psychiatry 1999;67:199-202. 
Jankowski L, Purson A, Teston B, Viallet F. Effets de la L-Dopa sur la dysprosodie et le fonctionnement laryngien de patients parkinsoniens. Actes des $\mathrm{XXV}^{\mathrm{e}}$ journées d'études sur la parole; 2004. p. 285-8.

Jiang J, Lin E, Wang J, Hanson DG. Glottographic measures before and after Levodopa treament in Parkinson's disease. Laryngoscope 1999;109:1287-94.

Johnson JA, Pring TR. Speech therapy and Parkinson'sdisease: a review and further data. Br J Disord Commun 1990;25: 183-94.

Kempler D, Van Lancker D. Effect of speech task on intelligibility in dysarthria: a case study of Parkinson's disease. Brain Lang 2002;80:449-64.

Kent RD, Rosenbek JC. Prosodic disturbance and neurologic lesions. Brain Lang 1992;15:259-91.

Kent RD, Kent JF, Weismer G, Duffy J. What dysarthrias can tell us about the neural control of speech? Journal of Phonetics 2000;28:273-302.

Klostermann F, Ehlen F, Vesper J, Nubel K, Gross M, Marzinzik F, et al. Effects of subthalamic deep brain stimulation on dysarthrophonia in Parkinson's disease. J Neurol Neurosurg Psychiatry 2008;79:522-9.

Liepmann H. Motor aphasia, anarthria and apraxia. Transaction of the XVIIth International Congress of Medicine in London; 1913. p. 97-106 [section xi, part 2].

Liotti M, Ramig LO, Vogel D, New P, Cook CI, Ingham RJ, et al. Hypophonia in Parkinson's disease: neural correlates of voice treatment revealed by PET. Neurology 2003;60:432-40.

Locco J. Aspects aérodynamiques de la production des occlusives dans la maladie de Parkinson. Actes des XXV journées d'études sur la parole; 2004. p. 317-20.

Logeman JA, Fisher HB, Boshes B, Blonsky ER. Frequency and cooccurrence of vocal tract dysfunctions in the speech of a large sample of Parkinson patients. J Speech Hear Disord 1978;43:47-57.

Luchsinger R, Arnold GE. Voice-speech-language: clinical communicology - its physiology and pathology. Belmont: Wadsworth Publishing Company; 1965.

Metter EJ, Hanson WR. Clinical and acoustical variability in hypokinetic dysarthria. J Commun Disord 1986;19:347-66.

Meunier C, Floccia C. Syllabe ou mot : quelle unité permet d'identifier les catégories phonétiques? Actes des journées d'études linguistiques; 1999. p. 87-92.

Monfrais-Pfauwadel MC. Palilalies et pseudo-bégaiements. In: Özsancak C, Auzou P, editors. Les troubles de la parole et de la déglutition dans la maladie de Parkinson. Marseille: Solal; 2005. p. 213-22.

Moreau C, Plachez A, Annic A, Pennel O, Viallet F, Pinto S, et al. Modulation of dysarthropneumophonia in advanced Parkinson's disease by low-frequency subthalamic nucleus deep brain stimulation. Mov Disord 2010;25(S2):S341.

Müller J, Wenning GK, Verny M. Progression of dysarthria and dysphagia in post-mortem-confirmed parkinsonian disorders. Arch Neurol 2001;58:259-64.

Narayana S, Jacks A, Robin DA, Poizner H, Zhang W, Franklin C, et al. A noninvasive imaging approach to understanding speech changes following deep brain stimulation in Parkinson's disease. Am J Speech Lang Pathol 2009;18: 146-61.

Narayana S, Fox PT, Zhang W, Franklin C, Robin DA, Vogel D, et al. Neural correlates of efficacy of voice therapy in Parkinson's disease identified by performance-correlation analysis. Hum Brain Map 2010;31:222-36.

Péran P, Rascol O, Démonet JF, Celsis P, Nespoulous JL, Dubois B, et al. Deficit of verb generation in nondemented patients with Parkinson's disease. Mov Disord 2003;18:150-6.

Pinto S, Gentil M, Fraix V, Benabib AL, Pollak P. Bilateral subthalamic stimulation effects on oral force control in Parkinson's disease. J Neurol 2003;250:179-87.
Pinto S, Özsancak C, Tripoliti E, Thobois S, Limousin-Dowsey P, Auzou P. Treatments for dysarthria in Parkinson's disease. Lancet Neurol 2004;3:547-56.

Pinto S, Thobois S, Costes N, Le Bars D, Benabid AL, Broussolle E, et al. Subthalamic nucleus stimulation and speech production in Parkinson's disease: a PET study. Brain 2004;127:602-15.

Pinto S, Gentil M, Krack P, Fraix V, Benabib AL, Pollak P. Changes induced by levodopa and subthalamic nucleus stimulation on parkinsonoan speech. Mov Disord 2005;20:1507-15.

Pitcairn TK, Clemie S, Gray JM, Pentland B. Impressions of parkinsonian patients from their recorded voices. $\mathrm{Br} \mathrm{J}$ Disord Commun 1990;25:85-92.

Poluha PC, Teulings HL, Brookshire RH. Handwriting and speech changes across the levodopa cycle in Parkinson's disease. Acta Psychol (Amst) 1998;100:71-84.

Ramig LO, Bonitati CM, Lemke JH, Horii Y. Voice treatment for patients with Parkinson's disease. Development of an approach and preliminary efficacy data. J Med Speech Lang Pathol 1994;2:191-209.

Ramig LO, Countryman S, Thompson L, Horii Y. A comparison of two forms of intensive speech treatment for Parkinson disease. J Speech Hear Res 1995;38:1232-51.

Ramig LO, Countryman S, O'Brien C, Hoehn M, Thompson L. Intensive speech treatment for patients with Parkinson disease: short and long-term comparison of two techniques. Neurology 1996;47:1496-504.

Ramig LO, Sapir S, Countryman S, Pawlas AA, O'Brien C, Hoehn $\mathrm{M}$, et al. Intensive voice treatment $\left(\mathrm{LSVT}^{\mathbb{R}}\right.$ ) for patients with Parkinson's disease: a 2 years follow up. J Neurol Neurosurg Psychiatry 2001;71:493-8.

Ramig LO, Sapir S, Fox C, Countryman S. Changes in vocal intensity following intensive voice treatment $\left(\mathrm{LSVT}^{\mathbb{R}}\right)$ in individuals with Parkinson disease: a comparison with untreated patients and normal age-matched controls. Mov Disord 2001;16:79-83.

Ramig LO, Fox C. Lee Silverman Voice Treatment. In: Auzou P, Rolland-Monnoury V, Pinto S, Özsancak C, editors. Les dysarthries. Marseille: Solal; 2007. p. 652-62.

Rektorova I, Barrett J, Mikl M, Rektor I, Paus T. Functional abnormalities in the primary orofacial sensorimotor cortex during speech in Parkinson's disease. Mov Disord 2007;22:2043-51.

Remacle M. Troubles de la voix. In: Rondal JA, Seron X, editors. Troubles du langage. Bases théoriques, diagnostic et rééducation. Sprimont, Belgique: Pierre Mardaga; 1999. p. 435-70.

Robert D, Spezza C. La dysphonie parkinsonienne et les troubles articulatoires dans la dysarthrie parkinsonienne. In: Özsancak C, Auzou P, editors. Les troubles de la parole et de la déglutition dans la maladie de Parkinson. Marseille: Solal; 2005. p. 131-59.

Robertson SJ, Thomson F. Speech therapy in Parkinson's disease: a study of the efficacy and long term effects on intensive treatment. Br J Disord Commun 1984;19:213-24.

Rolland-Monnoury V, Özsancak C. La prise en charge de la dysarthrie dans la maladie de Parkinson. In: Auzou P, Rolland-Monnoury V, Pinto S, Özsancak C, editors. Les dysarthries. Marseille: Solal; 2007. p. 675-83.

Rousseaux M, Krystkowiak P, Kozlowski O, Özsancak C, Blond S, Destee A. Effects of subthalamic nucleus stimulation on parkinsonian dysarthria and speech intelligibility. J Neurol 2004;251:327-34.

Sachin S, Senthil Kumaran S, Singh S, Goyal V, Shukla G, Mahajan $\mathrm{H}$, et al. Functional mapping in PD and PSP for sustained phonation and phoneme tasks. J Neurol Sci 2008;273:51-6. 
Sanabria J, Garcia Ruiz P, Guttierez R. The effect of Levodopa on vocal function in Parkinson's disease. Clin Neuropharmacol 2001;24:99-102.

Santens P, DeLetter M, Van Borsel J, De Reuck J, Caemaert J. Lateralized effects of subthalamic nucleus stimulation on different aspects of speech in Parkinson's disease. Brain Lang 2003;87:253-8.

Sapir S, Spielman J, Ramig L, Story B, Fox C. Effects of intensive voice treatment $\left(\mathrm{LSVT}^{\mathbb{B}}\right)$ on vowel articulation in dysarthric individuals with idiopathic parkinson disease: acoustic and perceptual findings. J Speech Lang Hear Res 2007;50:899-912.

Sarr MM, Pinto S, Jankowski L, Teston B, Purson A, Ghio A, et al. Contribution de la mesure de la pression intra-orale pour la compréhension des troubles de la coordination pneumophonique dans la dysarthrie parkinsonienne. Rev Neurol (Paris) 2009;165:1055-61.

Scott S, Caird F. Speech therapy for Parkinson's disease. J Neurol Neurosurg Psychiatry 1983;46:140-4.

Scott S, Caird F. The response of the apparent receptive speech disorder of Parkinson' disease to speech therapy. J Neurol Neurosurg Psychiatry 1984;47:302-4.

Selby G. Parkinson's disease. In: Vinken PJ, Bruyn GW, editors. Handbook of clinical neurology, vol. 6. Amsterdam: North Holland; 1968. p. 173-211.

Spielman J, Borod J, Ramig L. Effects of intensive voice treatment (LSVT) on facial expressivenes in Parkinson's disease: preliminary data. Cogn Behav Neurol 2003;16:177-88.

Teston B, Viallet F. La dysprosodie parkinsonienne. In: Özsancak C, Auzou P, editors. Les troubles de la parole et de la déglutition dans la maladie de Parkinson. Marseille: Solal; 2005. p. 161-93.

Tjaden K. An acoustic study of coarticulation in dysarthric speakers with parkinson's disease. J Speech Hear Res 2000;43:1466-80.
Törnqvist AL, Schalén L, Rehncrona S. Effects of different electrical parameters setting on the intelligibility of speech in patients with Parkinson's disease treated with subthalamic deep brain stimulation. Mov Disord 2005;20:416-23.

Tripoliti E, Zrinzo L, Martinez-Torres I, Tisch S, Frost E, Borrell $\mathrm{E}$, et al. Effects of contact location and voltage amplitude on speech and movement in bilateral subthalamic nucleus deep brain stimulation. Mov Disord 2008;23:2377-83.

Viallet F, Meynadier Y, Lagrue B, Teston B. The reductions of tonal range and of average pitch during speech production in « off " parkinsonians are restored by L-Dopa. Mov Disord 2000;15(S3):131.

Viallet F, Teston B, Jankowski L, Purson A. Effects of pharmacological versus electrophysiological treatments on parkinsonian dysprosody. Proceedings of speech prosody; 2002. p. 679-82.

Viallet F, Gayraud D. Les troubles de la production de parole au cours de la maladie de Parkinson : présentation générale. In: Özsancak C, Auzou P, editors. Les troubles de la parole et de la déglutition dans la maladie de Parkinson. Solal: Marseille; 2005. p. 99-109.

Viallet F, Teston B. La dysarthrie dans la maladie de Parkinson. In: Auzou P, Rolland-Monnoury V, Pinto S, Özsancak C, editors. Les dysarthries. Marseille: Solal; 2007. p. 375-82.

Yu P, Garrel R, Nicollas R, Ouaknine M, Giovanni A. Objective voice analysis in dysphonic patients: new data including nonlinear measurements. Folia Phoniatr Logop 2007;59(1):20-30.

Yuceturk AV, Yilmaz H, Egrilmez M, Karaca S. Voice analysis and videolaryngostroboscopy in patients with Parkinson's disease. Eur Arch Otorhinolaryngol 2002;259:290-3. 